\title{
Un método simplificado de predicción de hinchamiento de arcillas expansivas debido a cambios de humedad
}

\author{
A simplified method of swelling prediction for expansive clays due to moisture changes
}

Fecha de entrega: 3 de julio 2020

Fecha de aceptación: 7 de noviembre 2020

\section{Patricia C.A. Rodríguez}

Ferrara - Proyectos Especiales, Hannover 5686, Oficina 1140, La Reina, Chile, patricia.rodriguez@ferrara.cl

Los cambios volumétricos inducidos en suelos arcillosos expansivos debido a variaciones en su contenido de humedad han generado daños estructurales significativos en diversos países, y Chile no ha sido una excepción en este aspecto. Usualmente las primeras estimaciones del potencial de expansión se realizan en base a información relativamente limitada, previo al desarrollo de una campaña geotécnica más completa. Como alternativa a esta última se propone aquí un método sencillo que cuenta con una amplísima base experimental para predecir el hinchamiento de estas arcillas al aumentar su humedad, pudiendo incluso llegar a saturarse el suelo que es la situación eventual más crítica. A modo de ejemplo de utilización de este simple método se incluye en este artículo su aplicación al cálculo del movimiento vertical de una cimentación corrida, típica, en uno de los sitios investigados.

Palabras clave: arcillas expansivas, contenido de humedad, hinchamiento
Volumetric changes induced in expansive clays due to variations in their moisture content have originated significant structural damage in several countries. Chile has not been an exception in this matter. Preliminary assessments of these soils' potential volumetric changes is usually based on limited technical information before developing a complete geotechnical exploration. As an alternative to such estimations, a simple method based on extensive experimental data is herein proposed to predict the swelling potential of these clays when their moisture content increases, even until complete saturation of the soil, which is their most critical situation. An example of this simple method is its application to the calculation of the vertical movement of a typical strip foundation in one of the investigated sites.

Keywords: expansive clays, moisture content, swelling potential

\section{Introducción}

La expansión o contracción volumétrica experimentada por las arcillas debido a cambios de humedad ha sido causa de importantes daños estructurales en edificaciones y caminos, transformándose en un problema de enorme importancia práctica. La problemática de suelos expansivos se ha presentado en diversos países alrededor del mundo, tales como México, Estados Unidos, Australia, Sudáfrica, Turquía, Arabia Saudita, Inglaterra, España, Israel, Egipto e India (Abduljauwad et al., 1992; Juca et al., 1992; Carillo, 1969; Erguler y Ulusay, 2003; Sabtan, 2005; Rodríguez, 2007; Vanapalli y Lu, 2012; Puppala et al., 2013; Elbadry, 2016; Cantillo et al., 2017).
En Chile se ha detectado la presencia de arcillas expansivas en algunas comunas de la Región Metropolitana como Colina, Lo Barnechea, parte de Lampa, Las Condes, y Quilicura. En las ciudades de El Salvador (III Región), Malloa (VI Región), Talca (VII Región), y en los centros mineros de El Teniente y Chuquicamata (Rodríguez, 2007; Rodríguez-Roa, 1982). Dada su importancia en nuestro país, se encuentra actualmente en desarrollo la norma prNCh3608 (2020) sobre este tipo de suelos cuyo objetivo es dar a conocer esta problemática, incorporar algunas recomendaciones para la exploración geotécnica, y entregar soluciones de cimentaciones típicas. 
En la literatura técnica se encuentran diferentes criterios propuestos para evaluar el potencial expansivo de este tipo de suelos, principalmente en función de sus propiedades índice (Altemeyer, 1956; Holtz y Gibbs, 1956; Raman, 1967; Chen, 1975). Uno de los más usados ha sido el criterio propuesto por Holtz y Gibbs (1956), presentado en la Tabla 1.

Tabla 1: Clasificación de suelos expansivos según Holtz y Gibbs (1956)

\begin{tabular}{|c|c|c|c|}
\hline $\begin{array}{c}\text { Contenido } \\
\text { coloidal } \\
(\% \text { menor a } 1 \mu \mathrm{m})\end{array}$ & $\begin{array}{c}\text { Índice de } \\
\text { Plasticidad IP }\end{array}$ & $\begin{array}{c}\text { Límite de } \\
\text { Contracción } \\
\text { LC }\end{array}$ & $\begin{array}{c}\text { Grado de } \\
\text { expansión }\end{array}$ \\
\hline$>28$ & $>35$ & $<11$ & Muy alto \\
\hline $20-31$ & $25-41$ & $7-12$ & Alto \\
\hline $13-23$ & $15-28$ & $10-16$ & Medio \\
\hline$<15$ & $<18$ & $>15$ & Bajo \\
\hline
\end{tabular}

Estos criterios, si bien entregan una información de tipo cualitativa, no permiten cuantificar los movimientos esperados en el terreno. Para tal efecto, algunos autores han propuesto distintos métodos a fin de poder predecir la magnitud del hinchamiento (Lambe, 1960; Seed et al., 1962; Komornik y David, 1969; Vijayvergiya y Ghazzaly, 1973; Chen, 1975; Rao y Tripathy, 2003; Çimen et al., 2012). Sin embargo, dichos métodos han demostrado ser poco aplicables, puesto que algunos fueron generados en base a ensayos sobre probetas remoldeadas de arcillas, o bien no consideraron factores de vital importancia, como son la presión vertical aplicada por la cimentación y/o la humedad inicial del suelo. Otros, requieren ser complementados con ensayos previos de hinchamiento, consolidación, o mediciones de succión (Jiménez-Salas et al., 1980; Mckeen y Hamberg, 1981; Araya, 1993). Algunos métodos incorporan ensayos sobre muestras inalteradas como base para la calibración de sus modelos, además de requerir de parámetros difíciles de estimar en etapas tempranas de un proyecto, como son la succión de la matriz, información mineralógica de las arcillas presentes en el suelo, $\mathrm{pH}$, conductancia superficial o módulo de contracción-expansión (Aitchison, 1973; Johnson y Snethen, 1978; Mitchell y Avalle, 1984; Hamberg y Nelson, 1984; Dhowian, 1990; McKeen, 1992; Briaud et al., 2003; Ito y Hu, 2011; Lin y Cerato, 2012; Puppala et al., 2013).

Otros métodos basados también en ensayos sobre muestras inalteradas han incluido, además, variables relevantes asociadas al comportamiento de suelos expansivos que sean de fácil obtención en una caracterización inicial de suelos (Brackley, 1975; Rakela, 1987; Elbadry, 2016).

De todos estos métodos solamente el criterio propuesto por Rakela (1987) ha sido aplicado a suelos chilenos. Este criterio está limitado a aquellos suelos expansivos que se encuentran dentro de tres grupos definidos gráficamente, según su ubicación en la carta de plasticidad de Casagrande (Araya, 1993). Para cada grupo de suelos se propusieron curvas de hinchamiento diferentes en términos del nivel de saturación inicial del suelo y de la presión vertical aplicada. Esta consideración de grupos de suelos discretos, desconectados entre sí, impide su aplicación para el caso más general.

Se propone aquí un método general basado en la evidencia experimental, para predecir el hinchamiento de estratos de arcillas expansivas al saturarse. Situación eventual que es la condición más crítica que puede producirse durante la vida útil de una obra. A fin de ilustrar el uso de este método en la práctica profesional, se presenta aquí su aplicación al cálculo del movimiento vertical de una cimentación corrida, típica, en uno de los sitios investigados. En la presente investigación se han considerado los movimientos del terreno debidos solo a aumentos del contenido de humedad.

\section{Base experimental}

El programa experimental consistió, incialmente, en una serie de ensayos de clasificación de las muestras de arcilla estudiadas: límites de Atterberg, peso específico de las partículas sólidas, humedad, ensayos granulométricos, y granulometrías de finos o Bouyoucos. El comportamiento expansivo de estos suelos arcillosos frente a cambios en su contenido de humedad inicial, y/o en la carga aplicada, se estudió mediante ensayos de hinchamiento efectuados en edómetro, según la norma norma ASTM D4546 (1996). En dichas pruebas las probetas fueron sometidas a una presión vertical constante a lo largo de todo el ensayo.

La base experimental utilizada para generar y calibrar el modelo simplificado propuesto estuvo constituida por un total de 170 datos de ensayos de hinchamiento ejecutados sobre muestras no perturbadas procedentes de 11 estratos arcillosos diferentes. De los 170 ensayos, 75 fueron realizados específicamente para esta investigación (Rodríguez, 2007) y se identifican como Grupo A 
en la Tabla 2. Los restantes 95 datos de ensayos de hinchamiento corresponden a información obtenida de otras investigaciones nacionales (Araya, 1993; Queirolo, 1990; Rakela, 1987), identificadas como Grupo B en Tabla 2. En la Tabla 2 se observa, además, que las muestras consideradas en estos dos grupos de suelos fueron extraídas en diferentes sitios de la Región Metropolitana, excepto 27 ensayos (de los 95) efectuados sobre muestras provenientes de la ciudad de Talca.

En la presente investigación se incluyeron dos grupos adicionales de ensayos: Grupos C y D en la Tabla 3. En el Grupo C se recopilaron ensayos de hinchamiento realizados sobre suelos chilenos no utilizados en la calibración o ajuste del modelo propuesto, y en el Grupo D se recopilaron algunos ensayos de hinchamiento realizados en el extranjero, que tampoco se utilizaron en el proceso de calibración del modelo. El objetivo de incorporar la información reunida en los Grupos C y D fue evaluar la validación o capacidad de predicción del modelo.

Tabla 2: Base experimental utilizada en la generación (ajuste) del modelo propuesto

\begin{tabular}{|c|c|c|c|}
\hline Grupo & $\begin{array}{c}\mathrm{N}^{\mathrm{o}} \mathrm{de} \\
\text { ensayos de } \\
\text { hinchamiento }\end{array}$ & $\begin{array}{c}\text { Ubicación } \\
\text { (comuna, región) }\end{array}$ & $\begin{array}{c}\text { Fuente } \\
\text { (autor, año) }\end{array}$ \\
\hline A & 75 & $\begin{array}{c}\text { Colina, R.M. } \\
\text { Lampa, R.M. } \\
\text { Las Condes, R.M. }\end{array}$ & $\begin{array}{c}\text { Rodríguez, } \\
2007\end{array}$ \\
\hline B & 95 & $\begin{array}{c}\text { Quilicura, R.M. } \\
\text { Lampa, R.M. } \\
\text { Talca, Región del Maule }\end{array}$ & $\begin{array}{c}\text { Rakela, 1987 } \\
\text { Queirolo, 1990 } \\
\text { Araya, 1993 }\end{array}$ \\
\hline
\end{tabular}

Tabla 3: Base experimental utilizada para la validación del modelo propuesto

\begin{tabular}{|c|c|l|}
\hline Grupo & $\begin{array}{c}\mathrm{N}^{\mathrm{o}} \text { de } \\
\text { ensayos de } \\
\text { hinchamiento }\end{array}$ & \multicolumn{1}{c|}{$\begin{array}{c}\text { Ubicación } \\
\text { (país) }\end{array}$} \\
\hline $\mathrm{C}$ & 46 & Chile \\
\hline $\mathrm{D}$ & 36 & $\begin{array}{l}\text { Turquía } \\
\text { Arabia Saudita } \\
\text { Perú } \\
\text { Brasil }\end{array}$ \\
\hline
\end{tabular}

\section{Análisis preliminar de la evidencia empírica}

Los ensayos de hinchamento ejecutados sobre muestras inalteradas de arcillas reflejaron la existencia de una relación lineal entre el porcentaje de hinchamiento $\Delta H$ y el logaritmo de la presión vertical aplicada $P$, para un mismo nivel de saturación inicial, $S_{r}$ (Williams et al., 1985; Rodríguez, 2007):

$$
\Delta H(\%)=N-M \log \left(\frac{P}{P_{a}}\right)
$$

siendo $P_{\mathrm{a}}$ la presión atmosférica, $M$ y $N$ son dos parámetros auxiliares adimensionales. Las Figuras 1 a y $1 \mathrm{~b}$ muestran curvas características de hinchamiento versus presión aplicada, para un mismo valor de $S_{\mathrm{r}}$ obtenidas para el caso particular de dos de los sitios estudiados.

Por otra parte, para cada uno de los 11 estratos arcillosos estudiados, se encontró también una relación lineal entre los parámetros $M, N$ y el grado de saturación inicial, $S_{r}$, en función de cuatro parámetros adimensionales: $M_{m}, N_{m}, M_{n}$ y $N_{n}$, según se indica en las siguientes ecuaciones:

$$
\begin{aligned}
& M=M_{m} S_{r}(\%)+N_{m} \\
& N=M_{n} S_{r}(\%)+N_{n}
\end{aligned}
$$

Estos cuatro parámetros $M_{m}, N_{m}, M_{n}$ y $N_{n}$, resultaron ser constantes para cada estrato $u$ horizonte específico, pero variables de un estrato a otro, como se advierte en las Figuras 1c y 1d.

\section{Modelo propuesto para la predicción del hinchamiento}

Durante la presente investigación se observó, además, la estrecha relación que existe entre el porcentaje de hinchamiento de las arcillas al saturarse y su plasticidad y contenido de partículas finas (partículas de diámetro inferior a malla ASTM $\mathrm{N}^{\circ} 200$ ). Ello condujo a la conveniencia de corregir por porcentaje de finos la ecuación (1), de manera de poder obtener una expresión de carácter general más completa:

$$
\Delta H(\%)=\left[N^{*}-M^{*} \log \left(\frac{P}{P_{a}}\right)\right] \frac{\% \text { finos }}{100}
$$

donde, $M^{*}$ y $N^{*}$ son parámetros que dependen no sólo del grado de saturación inicial, sino, también, de la plasticidad de los finos contenidos en la arcilla. En efecto, en forma análoga a las ecuaciones (2) y (3), se ajustaron las relaciones:

$$
\begin{aligned}
& M^{*}=M_{m}{ }^{*} S_{r}(\%)+N_{m}{ }^{*} \\
& N^{*}=M_{n}{ }^{*} S_{r}(\%)+N_{n}{ }^{*}
\end{aligned}
$$

Teniendo en consideración la amplia base de datos experimentales recopilada, se examinaron distintas 
a)

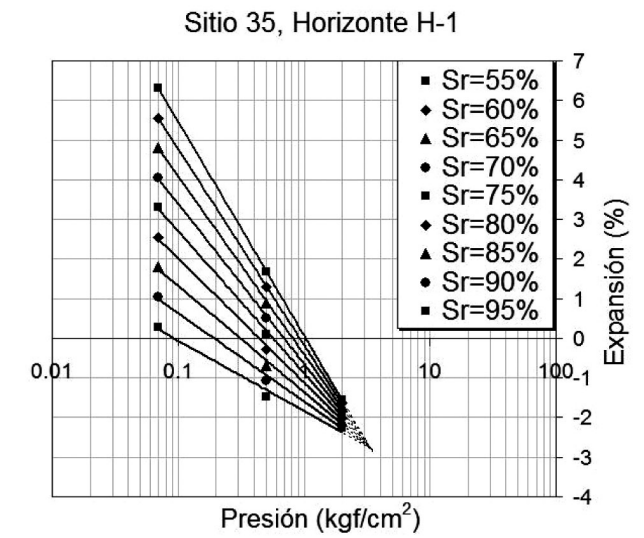

c)

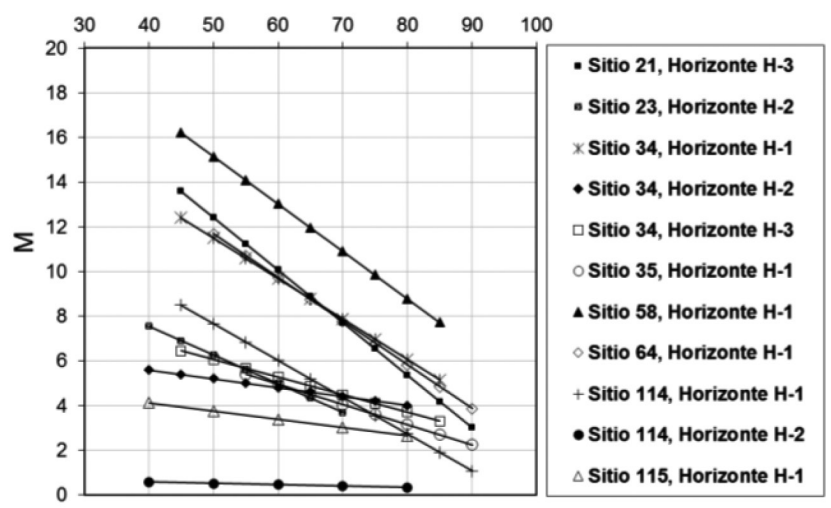

Saturación incial (\%)

Figura 1: Curvas de hinchamiento versus presión para diferentes valores de $S_{r}$ : a) sitio 35, H-1, grupo B, b) sitio 58, H-1, grupo A, c) y d) relaciones entre los factores $M$ y $N$ y el grado de saturación inicial $S_{r}$, datos grupos A y B

correlaciones estadísticas para el cálculo de los parámetros $M_{\mathrm{m}}{ }^{*}, N_{\mathrm{m}}{ }^{*}, M_{\mathrm{n}}{ }^{*}, \mathrm{y} N_{\mathrm{n}}{ }^{*}$. Las expresiones que arrojaron mejores resultados, y que complementan el modelo de predicción propuesto son:

$$
\begin{aligned}
& \log \left(N_{m}{ }^{*}\right)=-4.5269 \log \left(\frac{L L}{I P}\right)+2.0604 \\
& \log \left(-M_{m}{ }^{*}\right)=1.1028 \log \left(N_{m}{ }^{*}\right)-2.1929 \\
& \log \left(-M_{n}{ }^{*}\right)=1.1975 \log \left(-M_{m}{ }^{*}\right)+0.2387 \\
& N_{n}{ }^{*}=-102.43 M_{n}{ }^{*}-2.3487
\end{aligned}
$$

En las ecuaciones (8) y (9) los parámetros $M_{\mathrm{m}}{ }^{*}$ y $M_{\mathrm{n}}{ }^{*}$ resultan siempre con valores negativos. En la Figura 2 a se comparan los hinchamientos medidos con las predicciones entregadas por el modelo, incluyendo solamente los resultados de aquellos ensayos utilizados dentro del proceso de generación o calibración del mismo (capacidad de ajuste), mientras que en la Figura $2 \mathrm{~b}$ se presenta sólo la información derivada de ensayos que no fueron incorporados de ninguna forma en la generación del modelo (capacidad de predicción).
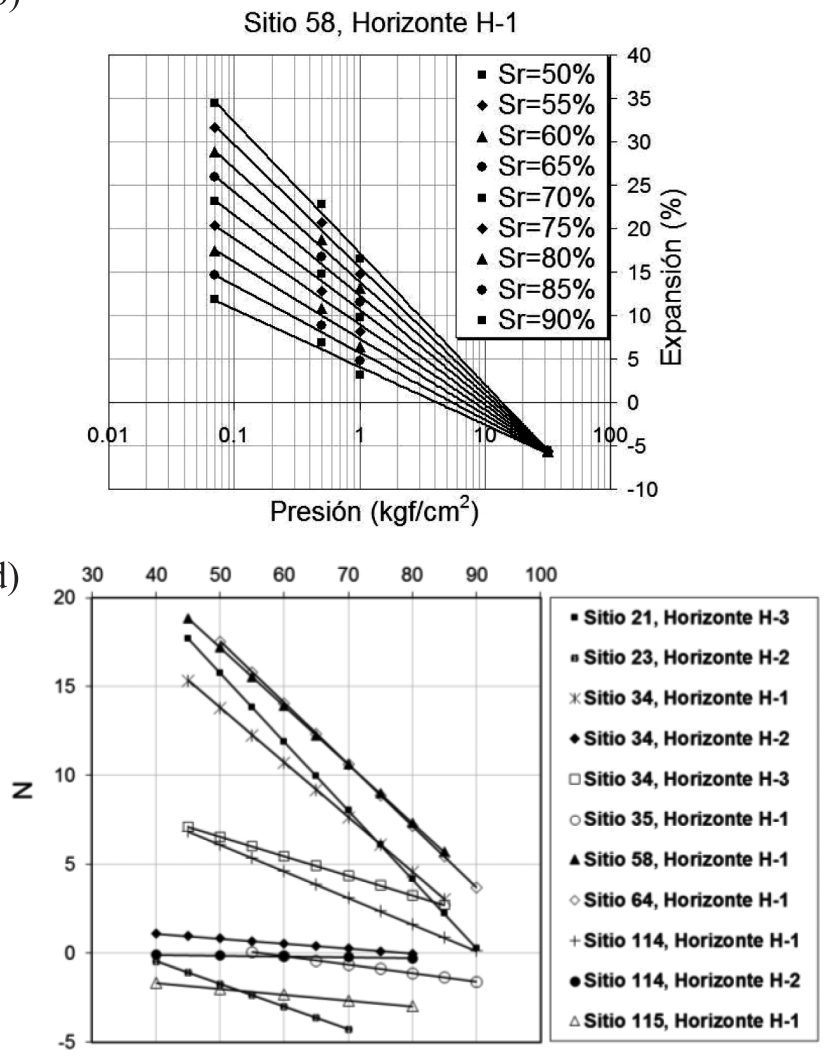

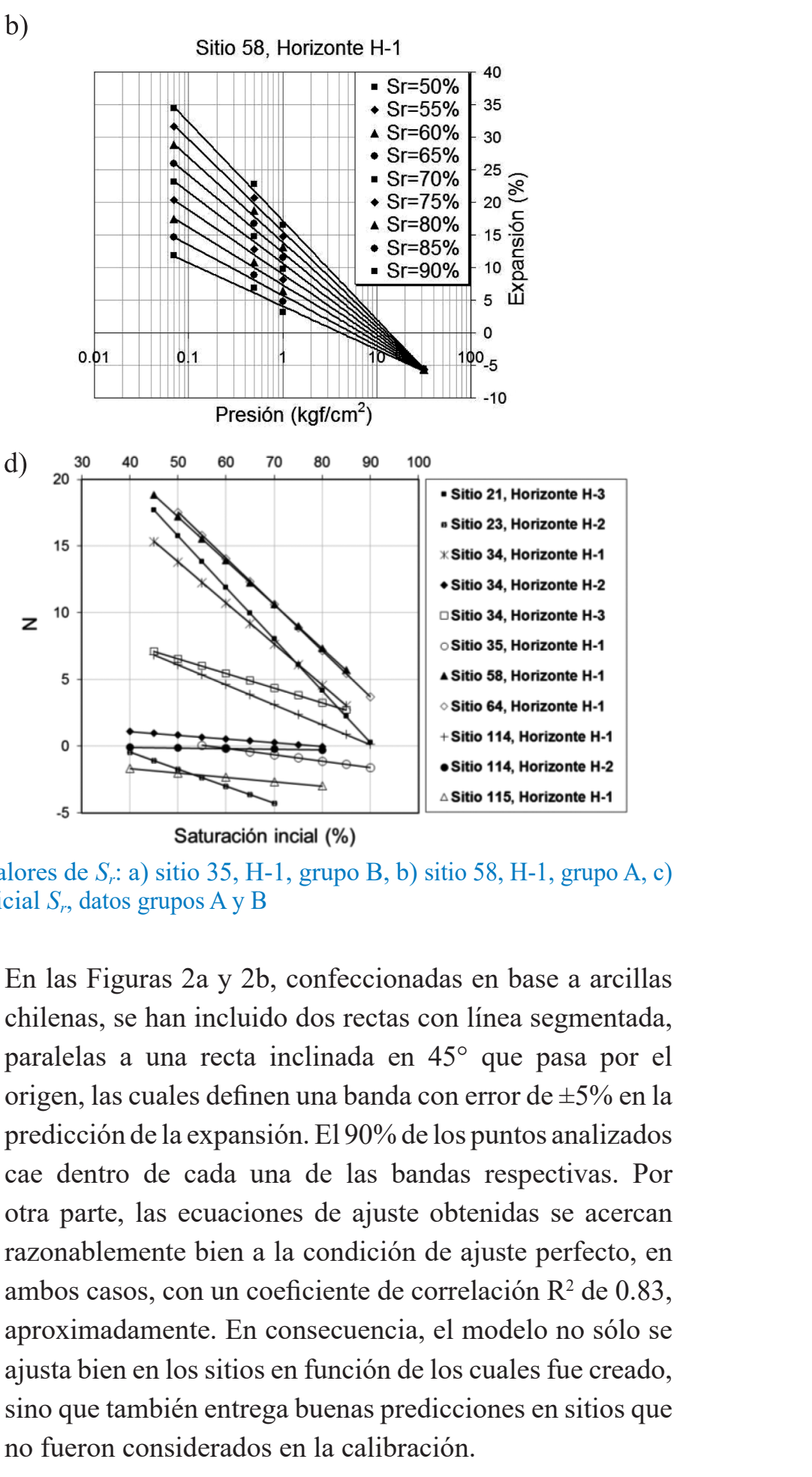

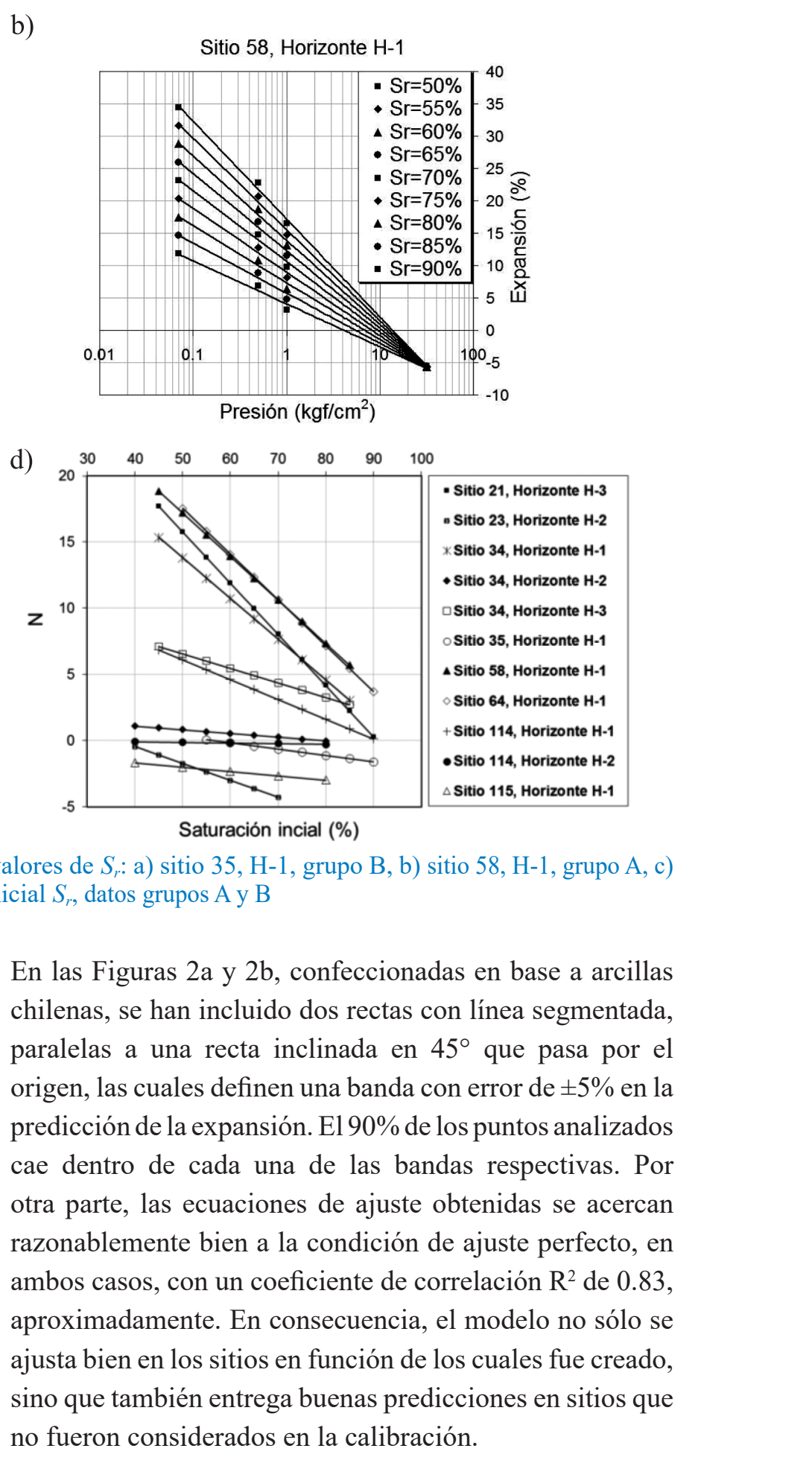

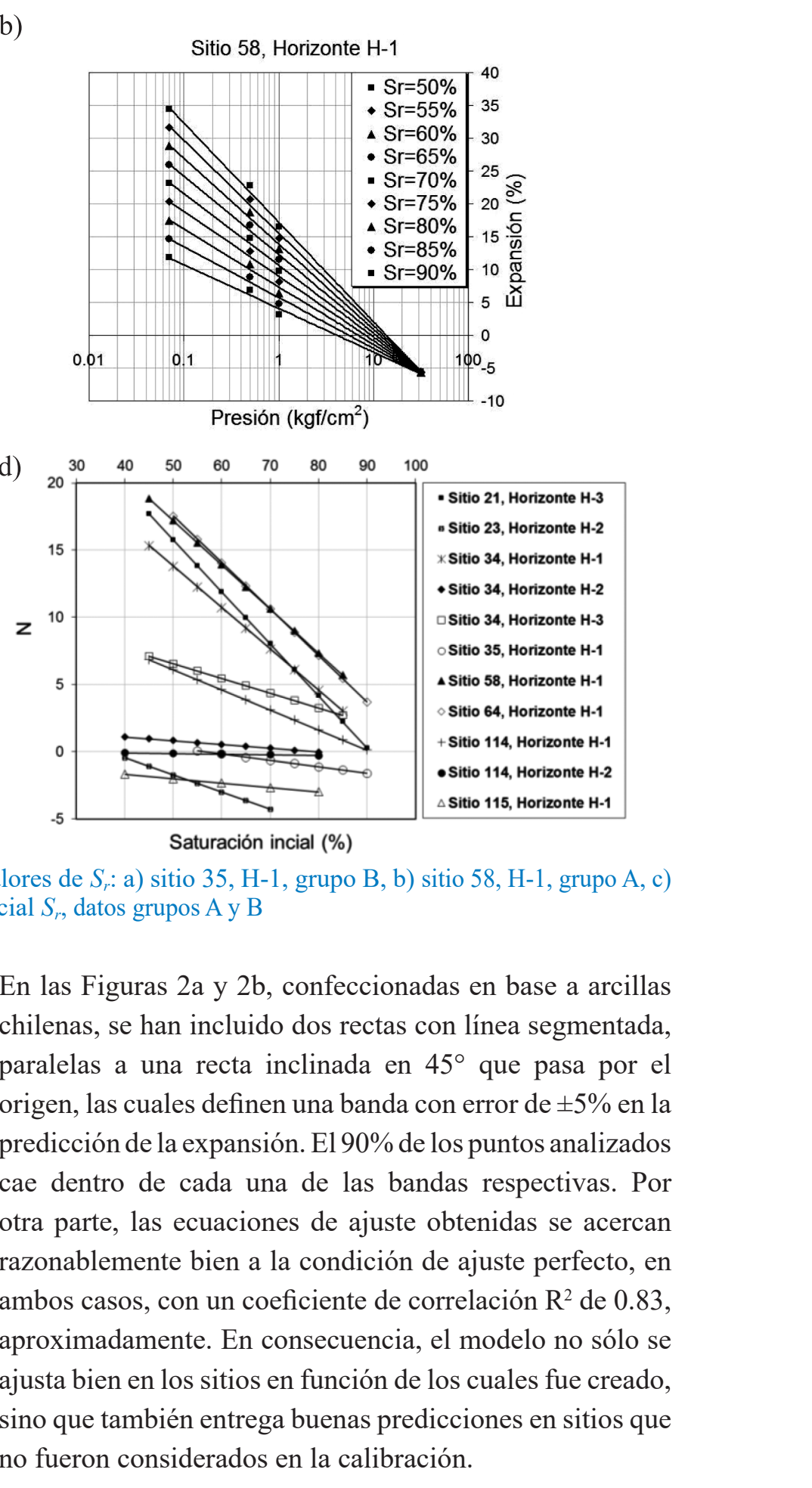

b)
$\begin{aligned} & \text { b) } \\ & \text { d) }\end{aligned}$
d)

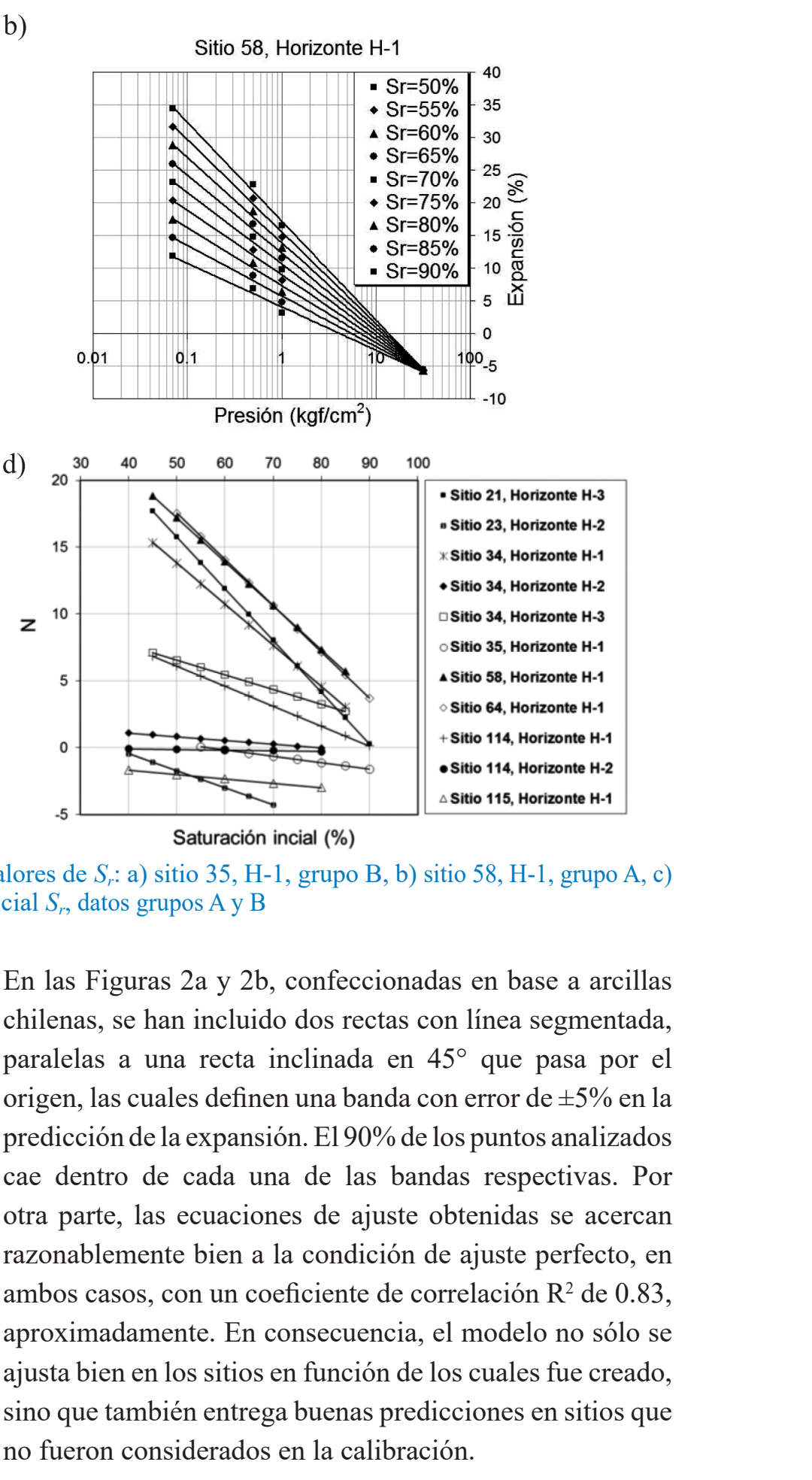

b)
$\begin{aligned} & \text { b) } \\ & \text { d) }\end{aligned}$
d)

b)
$\begin{aligned} & \text { b) } \\ & \text { d) }\end{aligned}$
d)

b)
$\begin{aligned} & \text { b) } \\ & \text { d) }\end{aligned}$
d)

b)
$\begin{aligned} & \text { b) } \\ & \text { d) }\end{aligned}$
d)

b)
$\begin{aligned} & \text { b) } \\ & \text { d) }\end{aligned}$
d)

b)
$\begin{aligned} & \text { b) } \\ & \text { d) }\end{aligned}$
d)

b)
$\begin{aligned} & \text { b) } \\ & \text { d) }\end{aligned}$
d)

b)
$\begin{aligned} & \text { b) } \\ & \text { d) }\end{aligned}$
d)

b)
$\begin{aligned} & \text { b) } \\ & \text { d) }\end{aligned}$
d)

b)
$\begin{aligned} & \text { b) } \\ & \text { d) }\end{aligned}$
d)

b)
$\begin{aligned} & \text { b) } \\ & \text { d) }\end{aligned}$
d) Con el objetivo de complementar la validación del modelo propuesto, se examinaron, además, ensayos de hinchamiento realizados sobre muestras inalteradas de arcillas extranjeras provenientes de Arabia Saudita (Abduljauwad et al., 1992; Sabtan, 2004), Turquía (Erguler y Ulusay, 2003), Brasil (Juca et al., 1992) y Perú (Carillo, 1969). Los resultados de este análisis se presentan en la 
a)

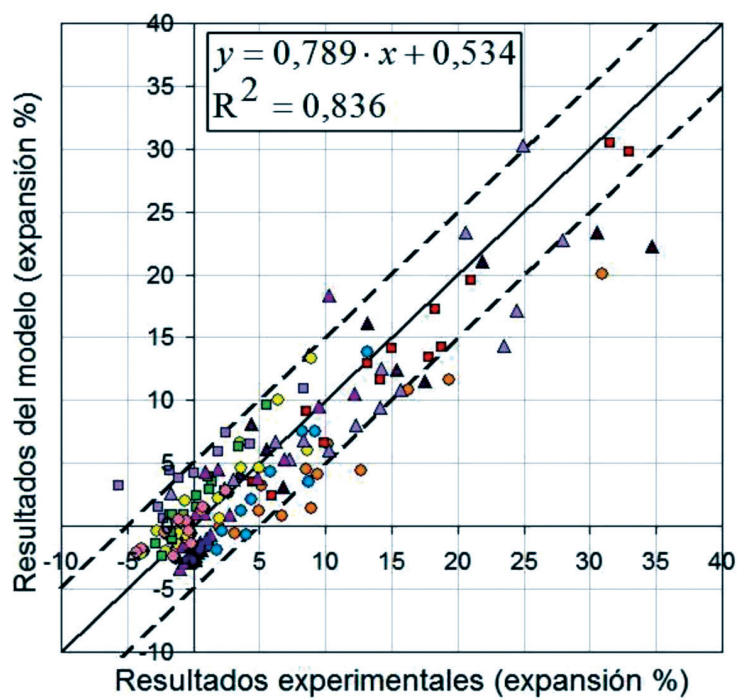

b)

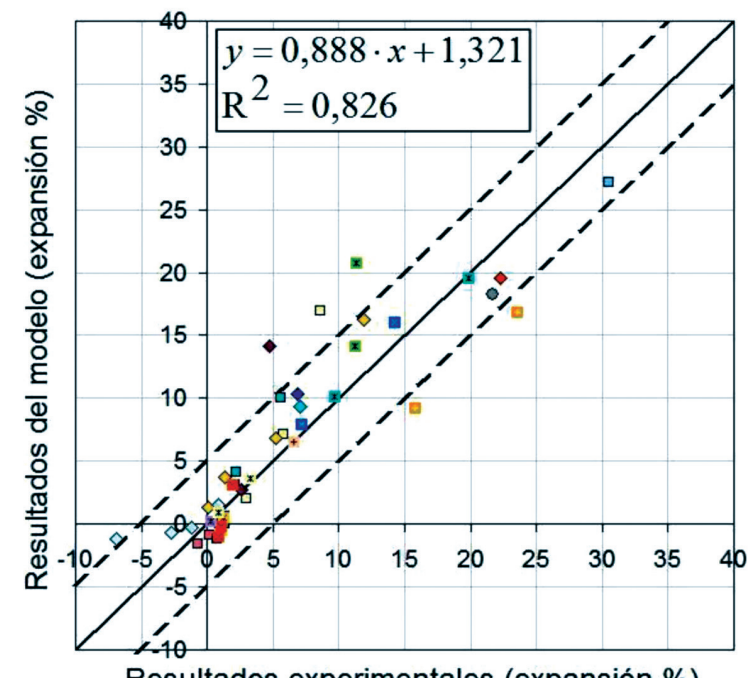

Figura 2: Validación del modelo propuesto en arcillas chilenas: a) capacidad de ajuste (datos de grupos A y B) y b) capacidad de predicción (datos de grupo C)

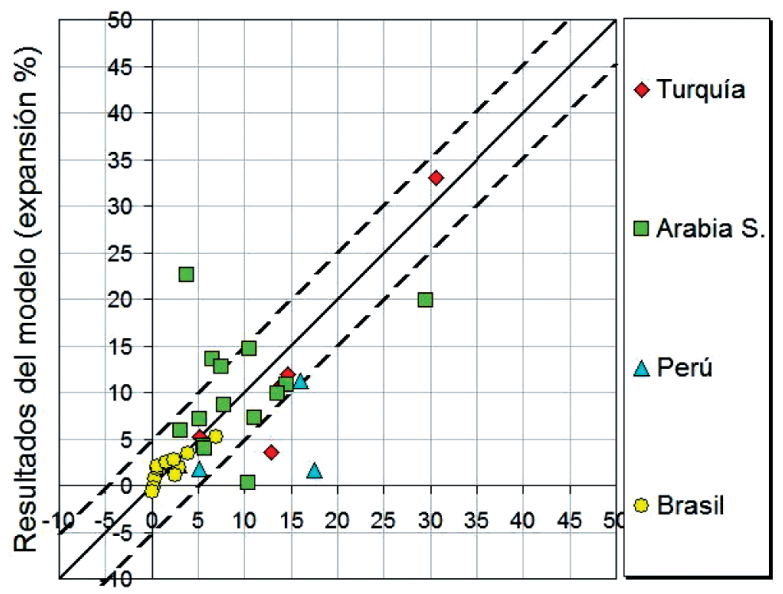

Resultados experimentales (expansión \%)

Figura 3: Verificación de la capacidad de predicción del modelo propuesto en arcillas extranjeras (datos del Grupo D)
Figura 3, en donde el $83 \%$ de los puntos considerados cae dentro de la banda con $\pm 5 \%$ de error en la predicción de la expansión.

En la Figura 4 se comparan las predicciones obtenidas mediante la aplicación de los criterios de Brackley (1975) y Rakela (1987), con las entregadas por el modelo propuesto para la totalidad de las arcillas chilenas consideradas en la presente investigación. Se observa que el criterio de Brackley (1975) arroja resultados que no reflejan en absoluto la tendencia de las arcillas chilenas. La razón de este resultado se debe a que dicho criterio fue generado a partir de arcillas sudafricanas que tendrían propiedades muy particulares, y por tanto sólo sería aplicable a esa zona. Lamentablemente, no se dispone de mayores antecedentes al respecto. Con relación al criterio de Rakela (1987), cabe señalar que éste entrega una predicción algo mejor, pero de calidad significativamente inferior al modelo aquí propuesto.

Por otro lado, la literatura también provee algunas propuestas de predicciones utilizando redes neuronales (Das et al., 2010; Erzin y Güneş, 2011; Yilmaz y Kaynar, 2011). Por lo anterior, de forma complementaria se llevó a cabo, además, una comparación del modelo propuesto con las predicciones entregadas por programas computacionales basados en redes neuronales. Los programas de simulación basados en redes neuronales son modelos de aprendizaje y procesamiento automático inspirados en el sistema nervioso humano. En ellos, el aprendizaje se materializa mediante una serie de factores que son ajustados en función de la información ingresada en la red, de modo que en una red neuronal entrenada, el conjunto de factores determina el conocimiento de dicha red y tiene la propiedad de resolver el problema para el que ella ha sido entrenada. La aproximación iterativa de los factores que permiten determinar la función de ajuste en cada punto, en la mayoría de los programas de redes neuronales disponibles, opera con el algoritmo Backpropagation, el cual fue también utilizado en las simulaciones realizadas en esta investigación. Se usaron dos programas computacionales: el BrainCom, y el Toolbox Neural Networks incluido en la versión 6.5.1 de Matlab.

El entrenamiento previo de la red requerido para la simulación, se llevó a cabo utilizando solamente la parte de la base de datos de las arcillas chilenas usada para la confección de la Figura 2a. La capacidad de ajuste obtenida 

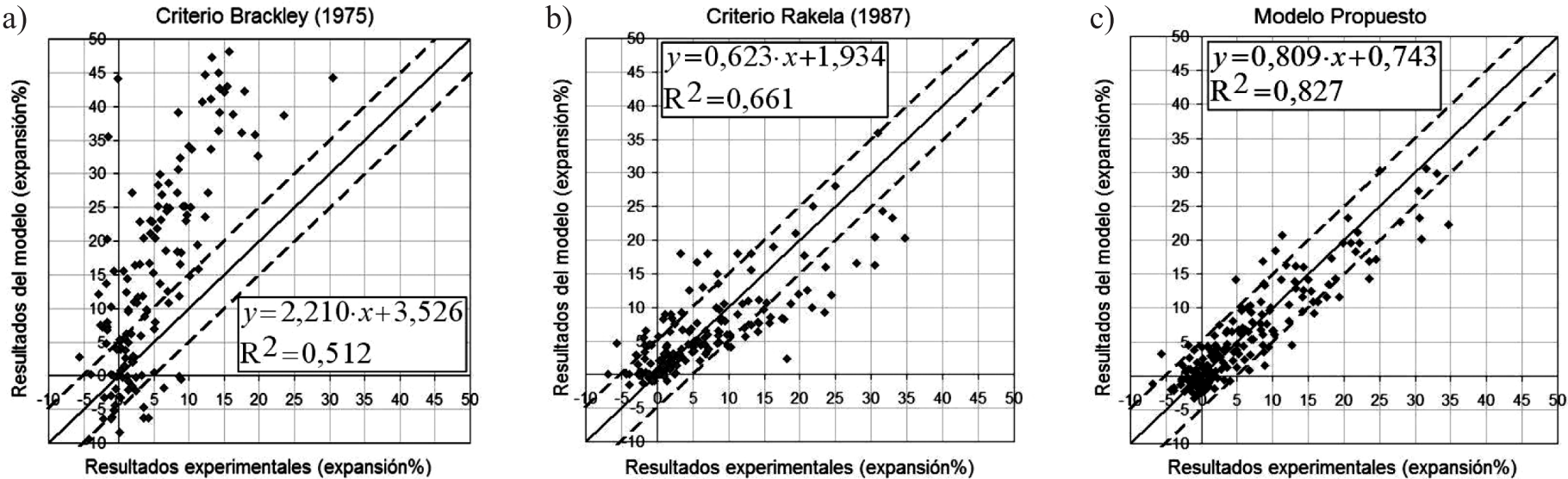

Figura 4: Aplicación del modelo propuesto y de los criterios de Brackley (1975) y Rakela (1987), a la totalidad de las arcillas chilenas consideradas (datos de grupos A, B, C y D)

a)

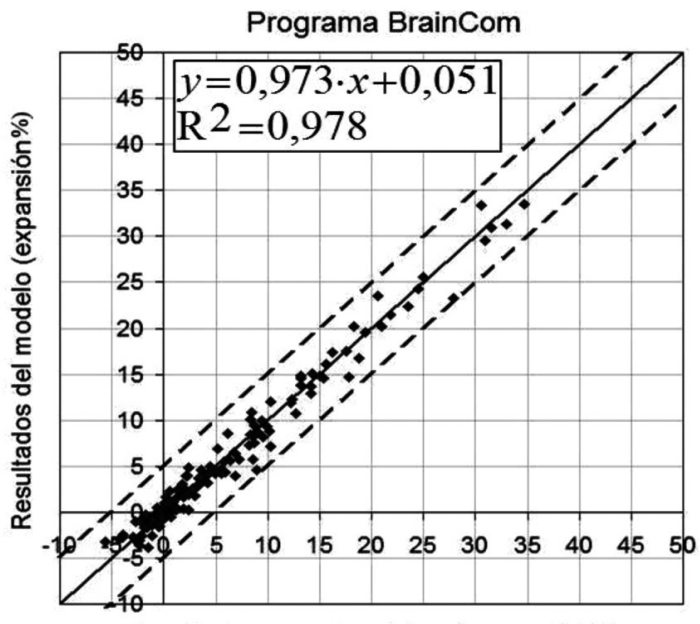

Resultados experimentales (expansión\%)

Programa BrainCom

c)

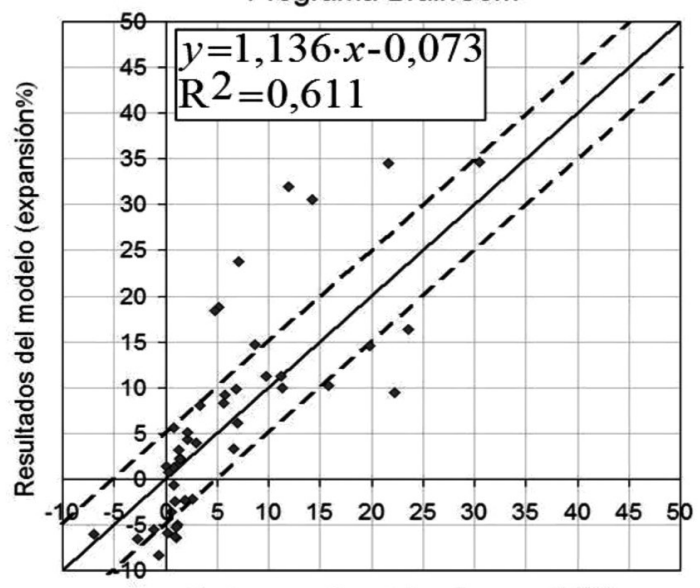

Resultados experimentales (expansión\%) b)

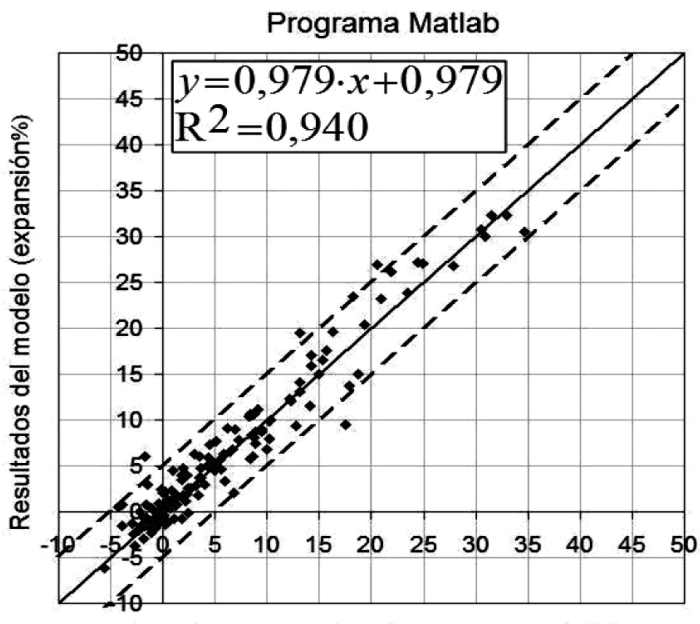

Resultados experimentales (expansión\%)

d)

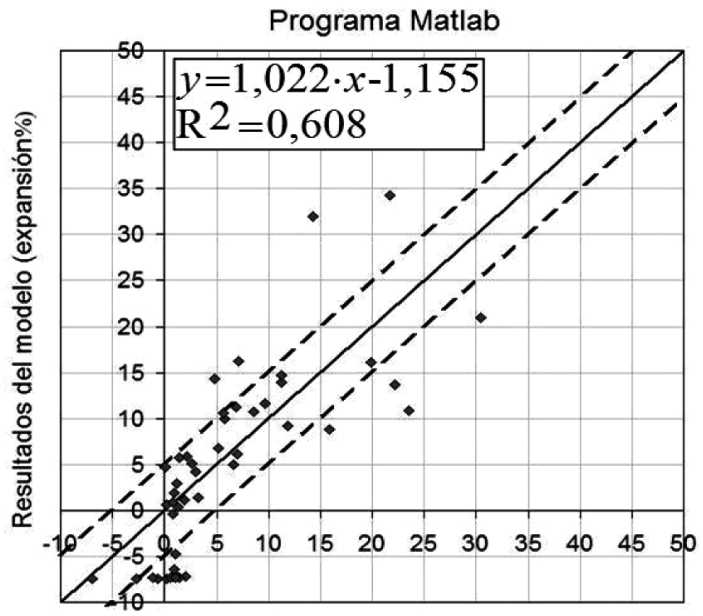

Resultados experimentales (expansión\%)

Figura 5: Resultados obtenidos con programas basados en redes neuronales. Capacidad de ajuste para arcillas chilenas (datos grupos A y B) con el programa: a) BrainCom y b) Matlab. Capacidad de predicción para arcillas chilenas (datos grupo C) con el programa: c) BrainCom y d) Matlab

con los programas BrainCom y Matlab se muestra en las Figuras $5 \mathrm{a}$ y $5 \mathrm{~b}$, respectivamente. Se observa en estas figuras que el coeficiente de correlación $\mathrm{R}^{2}$ varió entre 0.940 y 0.978 , contra un valor de 0.836 alcanzado con el modelo propuesto, según se indica en la Figura 2a. Sin embargo, al considerar las arcillas chilenas no incluidas en la base de datos empleada para la calibración o entrenamiento de los programas, su capacidad de predicción resulta muy 
inferior a la entregada por el modelo propuesto, como se infiere de la comparación de los valores del coeficiente $\mathrm{R}^{2}$ incluidos en las Figuras $5 \mathrm{c}, 5 \mathrm{~d}$, y 2b. La explicación de estos resultados está ligada al hecho de que las redes neuronales son incapaces de identificar o seleccionar dentro de su proceso de aprendizaje, los conceptos y variables realmente relevantes que gobiernan el fenómeno en cuestión.

\section{Aplicación del modelo propuesto a un caso típico de cimentación corrida}

Se ha considerado una zapata corrida de $60 \mathrm{~cm}$ de ancho que transmite una carga de $100 \mathrm{kPa}\left(1 \mathrm{~kg} / \mathrm{cm}^{2}\right)$ a nivel del sello de cimentación. El suelo de fundación es un estrato de arcilla de alta plasticidad, con $L L=72, I P=53$ y un porcentaje de finos de $81.41 \%$. Para este suelo, los parámetros correspondientes a ecuaciones (7) a (10) son los siguientes: $N_{\mathrm{m}}^{*}=28.712, M_{\mathrm{m}}^{*}=-0.260, M_{\mathrm{n}}^{*}=-0.345$ y $N_{\mathrm{n}}^{*}=33.023$.

En la aplicación que se presenta en este artículo se consideró la posibilidad de que, con posterioridad a la construcción de la vivienda, se pudiera saturar el suelo situado bajo la cimentación y alrededor de ella, producto de la infiltración de aguas lluvias en el terreno y/o por riego de jardines. En el sitio de aplicación del modelo propuesto no hay napa freática, y el grado de saturación inicial existente se aproximó a una variación lineal con la profundidad entre $z=0 \mathrm{~m} \mathrm{y} z=1.5 \mathrm{~m}$, según el perfil hídrico indicado en la Figura 6a. A partir de $1.5 \mathrm{~m}$ de profundidad, se supuso que el contenido de humedad se conserva aproximadamente constante.

Para la evaluación del levantamiento de la cimentación se discretizó el suelo subyacente en una serie de capas de espesor constante, y se calculó en cada una de ellas el hinchamiento experimentado en función de su condición inicial de saturación, y de la carga vertical recibida debida a la sobrecarga efectiva de suelo y de la presión transmitida por la estructura. Los incrementos de tensiones, $\Delta q$, en el plano medio de cada capa, debidos a la presión transmitida por la cimentación, se calcularon mediante la teoría de elasticidad (Figura 6b).

La Figura 6c presenta los resultados obtenidos para el levantamiento de la cimentación versus la profundidad saturada del estrato arcilloso, para los distintos espesores a)

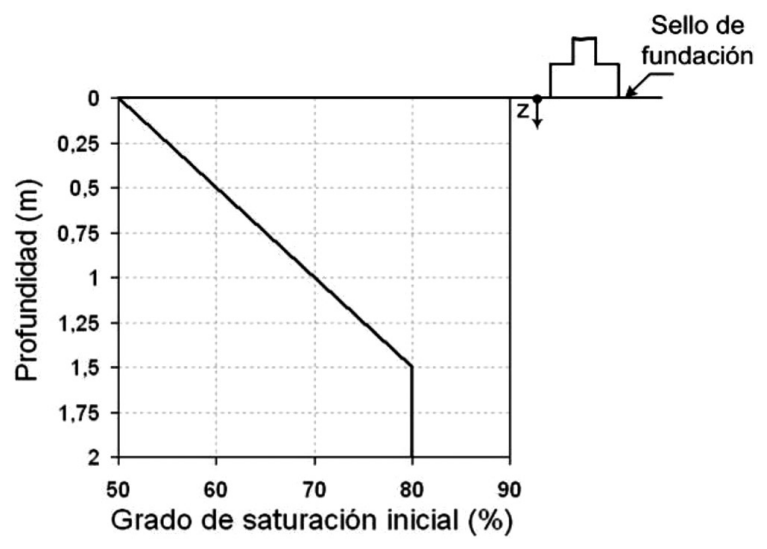

b)

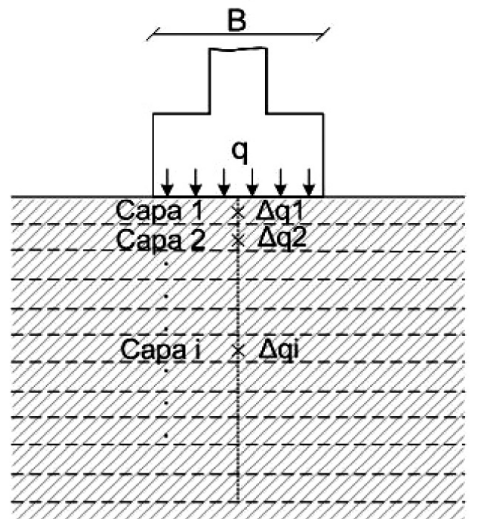

c)

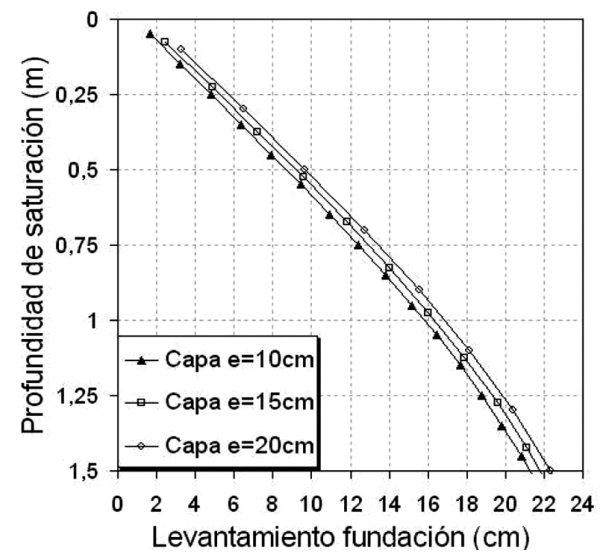

d)

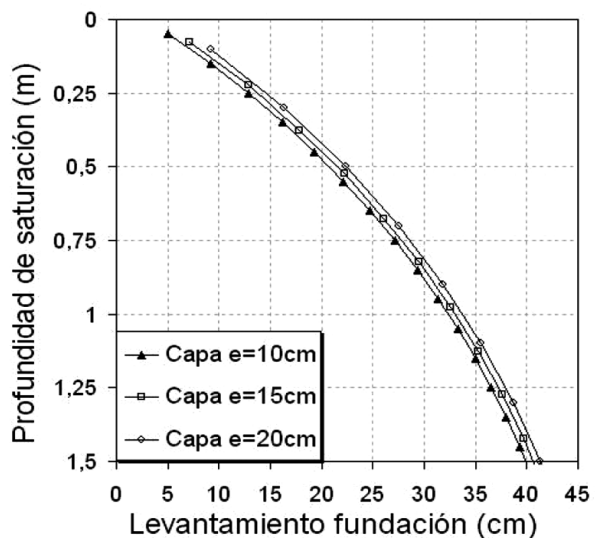

Figura 6: a) Perfil hídrico inicial adoptado para el cálculo del levantamiento de la cimentación, b) esquema de cálculo considerado para el análisis del levantamiento, c) levantamiento del terreno bajo la zapata corrida y d) levantamiento del terreno en zona sin cimentación 
de capa, e, considerados. Como era de esperar, el levantamiento aumenta con el espesor de arcilla saturada, registrándose un valor máximo de $22 \mathrm{~cm}$ para un espesor saturado de $1.5 \mathrm{~m}$. Resultados que ilustran claramente los enormes daños potenciales que podrían generarse en estructuras cimentadas en este tipo de suelos, en caso de no adoptarse las medidas adecuadas para mitigar o erradicar estos efectos.

En la Figura 6d se han incluido los levantamientos obtenidos para el caso de superficie libre, es decir, cuando no existe carga alguna en la cota $z=0 \mathrm{~m}$. En este caso, los levantamientos del terreno prácticamente doblan al valor del levantamiento máximo calculado anteriormente. De los resultados alcanzados en las dos aplicaciones efectuadas (Figuras 6c y 6d) se concluye que, para todos los efectos prácticos, es suficientemente preciso calcular el levantamiento del terreno subdividiendo el estrato de suelo que podría saturarse, en capas de altura del orden del $10 \%$ de su espesor.

\section{Conclusiones}

La presencia de estratos de suelos potencialmente expansivos puede resultar en graves problemas a las estructuras influenciadas por ellos. Si bien el proceso de hinchamiento por saturación de suelos expansivos es complejo y depende de muchos parámetros, la necesidad de realizar estimaciones ajustadas en etapas preliminares de un proyecto hace necesario el uso de parámetros simples, para realizar una primera aproximación del nivel de deformación esperada en un estrato de suelo expansivo, según el nivel de saturación inicial en que se encuentre y la solicitación axial a que se verá sometido.

Para atender a la necesidad antes descrita, a partir del análisis de 170 ensayos de hinchamiento de muestras de arcillas no perturbadas, se propone un modelo simplificado de predicción que ha demostrado tener un excelente ajuste a la evidencia experimental, tanto en arcillas chilenas como extranjeras. Este modelo requiere conocer únicamente parámetros simples y de fácil obtención como son el Límite Líquido, el Índice de Plasticidad, el Porcentaje de Finos, el grado de saturación inicial del suelo, y la carga aplicada por la cimentación. La mayor ventaja del modelo propuesto, además de su calidad de ajuste experimental, es que utiliza parámetros que se encuentran disponibles incluso en etapas tempranas de una campaña de exploración geotécnica, permitiendo realizar estimaciones en fases iniciales de un proyecto.

Como ejemplo de aplicación del modelo propuesto, se presenta el cálculo del levantamiento de una cimentación corrida, típica, apoyada sobre una arcilla de alta plasticidad, obteniéndose un levantamiento máximo de $22 \mathrm{~cm}$ al saturarse un espesor de suelo de $1.5 \mathrm{~m}$. Este valor prácticamente se duplicó en el caso de la superficie libre analizada. Levantamientos del terreno que ilustran claramente los enormes daños potenciales que podrían generarse en estructuras cimentadas en este tipo de suelos, en caso de no adoptarse las medidas adecuadas para mitigar o erradicar estos efectos.

\section{Agradecimientos}

La autora desea agradecer al Profesor Dr. Fernando Rodríguez-Roa, Profesor Emérito del Departamento de Ingeniería Estructural y Geotécnica de la Pontificia Universidad Católica de Chile, por sus valiosos aportes a esta investigación y al trabajo conjunto y colaborativo que dio origen a este artículo.

\section{Referencias}

Abduljauwad, S.N., Hameed, R.A., Al-Sulaimani, G.J., Basunbul, I.A. and Safar M.M. (1992). Expansive soils in eastern Province of Saudi Arabia. $7^{\text {th }}$ International Conference on Expansive Soils, Dallas, USA, 426-431

Aitchison, G.D. (1973). A quantitative description of the stressdeformation behavior of expansive soils. $3^{\text {rd }}$ International Conference on Expansive Soils, Haifa, Israel, vol. 2, 79-82

Altemeyer, W.T. (1956). Discussion of Engineering properties of expansive clays. Transactions of the American Society of Civil Engineers 121(1), 666-669

Araya, C.O. (1993). Estudio del comportamiento de suelos finos de Santiago y de otras zonas problemas. Memoria de título de Ingeniero Civil, Universidad de Chile, Santiago, Chile

ASTM D4546 (1996). Standard test methods for one-dimensional swell or settlement potential of cohesive soils. American Society for Testing and Materials ASTM, West Conshohocken, USA Brackley, I.J.A. (1975). The interrelationship of the factors affecting heave of an expansive unsaturated soil. $\mathrm{PhD}$ thesis, University of Natal, Durban, South Africa 
Briaud, J.L., Zhang, X. and Moon, S. (2003). Shrink test-water content method for shrink and swell predictions. Journal of Geotechnical and Geoenvironmental Engineering 129(7), 590-600

Cantillo, V., Mercado, V. and Pájaro, C. (2017). Empirical correlations for the swelling pressure of expansive clays in the city of Barranquilla, Colombia. Earth Sciences Research Journal 21(1), 45-49

Carillo, A. (1969). Contribution to the study of expansive clays of Peru. Second International Research and Engineering Conference on Expansive Clay Soils, Texas, USA, 183-193

Çimen, Ö., Keskin, S.N. and Yıldırım, H. (2012). Prediction of swelling potential and pressure in compacted clay. Arabian Journal for Science and Engineering 37(6), 1535-1546

Chen, F.H. (1975). Foundations on expansive soils. Elsevier, Amsterdam, The Netherlands

Das, S.K., Samui, P., Sabat, A.K. and Sitharam, T.G. (2010). Prediction of swelling pressure of soil using artificial intelligence techniques. Environmental Earth Sciences 61(2), 393-403

Dhowian, A.W. (1990). Field performance of expansive shale formation. JKAU Engineering Sciences 2,165-182

Elbadry, H. (2017). Simplified reliable prediction method for determining the volume change of expansive soils based on simply physical tests. HBRC journal 13(3), 353-360

Erguler, Z.A. and Ulusay, R. (2003). A simple test and predictive models for assessing swell potential of Ankara (Turkey) clay. Engineering Geology 67, 331-352

Erzin, Y. and Güneş, N. (2011). The prediction of swell percent and swell pressure by using neural networks. Mathematical and Computational Applications 16(2), 425-436

Hamberg, D.J. and Nelson, J.D. (1984). Prediction of floor slab heave. Fifth International Conference on Expansive Soils, Adelaide, Australia, 137-140

Holtz, W.G. and Gibbs, H.J. (1956). Engineering properties of expansive clays. Transactions of the American Society of Civil Engineers 121(1), 641-663

Ito, M. and Hu, Y. (2011). Prediction of the behaviour of expansive soils. $14^{\text {th }}$ Pan-American Conference on Soil Mechanics and Geotechnical Engineering, Toronto, Canada, 1-8
Jiménez-Salas, J.A., De Justo Alpañez, J.L. y Serrano González, A.A. (1980). Geotecnia y Cimientos III, Cimentaciones, Excavaciones y Aplicaciones de la Geotecnia. Editorial Rueda, Madrid, España

Johnson, L.D. and Snethen, D.R. (1978). Prediction of potential heave of swelling soil. Geotechnical Testing Journal 1(3), 117-124 Juca, J.F.T., Gusmao Filho, J.A. and Justino da Silva, J.M. (1992). Laboratory and field tests on an expansive soil in Brazil. $7^{\text {th }}$ International Conference on Expansive Soils, Dallas, USA, 337-342

Komornik, A. and David, D. (1969). Prediction of swelling pressure of clays. Journal of the Soil Mechanics and Foundation Division 95(1), 209-226

Lambe, T.W. (1960). The character and identification of expansive soils, soil PVC meter. Technical Studies Program, Federal Housing Administration, FHA 701, Washington DC, USA

Lin, B. and Cerato, A.B. (2012). Prediction of expansive soil swelling based on four micro-scale properties. Bulletin of Engineering Geology and the Environment 71(1), 71-78

McKeen, R.G. (1992). A model for predicting expansive soil behavior. 7th International Conference on Expansive Soils, Dallas, USA, 1-6

Mckeen, R.G. and Hamberg, D.J. (1981). Characterization of expansive soils. Transportation Research Record 790, 73-78

Mitchell, P.W. and Avalle, D.L. (1984). A technique to predict expansive soil movements. Fifth International Conference on Expansive Soils, Adelaide, Australia, 124-130

prNCh3608 (2020). Suelo expansivo - Requisitos geotécnicos para diseño y ejecución de obras de edificación y urbanización. Instituto Nacional de Normalización INN, Santiago, Chile

Puppala, A.J., Manosuthikij, T. and Chittoori, B.C. (2013). Swell and shrinkage characterizations of unsaturated expansive clays from Texas. Engineering Geology 164, 187-194

Queirolo, F.J. (1990). Estudio del comportamiento de algunas arcillas expansivas de Talca y Quilicura. Memoria de título de Ingeniero Civil, Universidad de Chile, Santiago, Chile 
Rakela, M. (1987). Estudio del comportamiento de las arcillas expansivas de Santiago. Memoria de título de Ingeniero Civil, Universidad de Chile, Santiago, Chile

Raman, V. (1967). Identification of expansive soils from the plasticity index and the shrinkage index data. The Indian Engineer 11(1), 17-22

Rao, K.S. and Tripathy, S. (2003). Effect of aging on swelling and swell-shrink behavior of a compacted expansive soil. Geotechnical Testing Journal 26(1), 36-46

Rodríguez-Roa, F. (1982). Fundaciones en arcillas expansivas. Primer Congreso Chileno de Ingeniería Geotécnica, Santiago, Chile, 355-382

Rodríguez, P.C.A. (2007). Predicción de los movimientos en cimentaciones construidas sobre arcillas expansivas debido a cambios de humedad. Tesis de Magíster, Pontificia Universidad Católica de Chile, Santiago, Chile

Sabtan, A.A. (2005). Geotechnical properties of expansive clay shale in Tabuk, Saudi Arabia. Journal of Asian Earth Sciences 25(5), 747-757
Seed, H.B., Woodward, R. and Lundgren, R. (1962). Prediction of swelling potential for compacted clays. Journal of the Soil Mechanics and Foundations Division 88(3), 53-87

Vanapalli, S. and Lu, L. (2012). A state-of-the art review of 1-D heave prediction methods for expansive soils. International Journal of Geotechnical Engineering 6(1), 15-41

Vijayvergiya, V.N. and Ghazzaly, O.I. (1973). Prediction of swelling potential for natural clays. Third International Conference on Expansive Soils, Haifa, Israel, vol. 1, 227-236

Williams, A.A.B., Pidgeon, J.T. and Day, P.W. (1985). Expansive soils. The Civil Engineer in South Africa 27(7), 367-378

Yilmaz, I. and Kaynar, O. (2011). Multiple regression, ANN (RBF, MLP) and ANFIS models for prediction of swell potential of clayey soils. Expert Systems with Applications 38(5), 59585966 